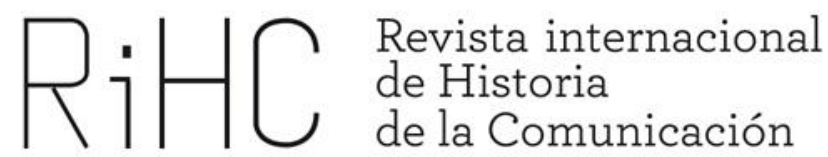

\title{
AMARILLISMO E INFOENTRETENIMIENTO EN LA INFORMACIÓN TELEVISIVA SOBRE LA CRISIS ECONÓMICA: EL CASO DE CUATRO TV (2012)
}

DOI: http://dx.doi.org/10.12795/RiHC.2015.i05.05

Francisco Segado-Boj

Universidad Internacional de la Rioja

Francisco.segado@unir.net

Recibido: 22-9-2014

Aceptado: 19-1-2015

Resumen:Este artículo mide el impacto del infoentretenimiento y el amarillismo televisivo en las noticias sobre la crisis económica emitidas por Cuatro TV entre abril y junio de 2012. Para ello aplica un análisis de contenido a una selección aleatoria de noticias ( $n=159)$ en el que se consideran las siguientes variables: piezas emitidas sobre la crisis, duración de las mismas, posición que ocupan en la escaleta de emisión, encuadre aplicado en las mismas y tipo de fuentes que aparecen en las informaciones. Se concluye que el principal rasgo sensacionalista presente en estas noticias es la preferencia por fuentes de tipo personal e individual, mientras que la adopción del enfoque del interés humano es secundario. 
Palabras clave: Televisión, crisis económica, sensacionalismo, infoentretenimiento, interés humano, personalización

\begin{abstract}
This paper measures the impact of infotainment and sensationalism on television news dealing with the economic crisis broadcasted by the Spanish network Cuatro TV between april and june, 2012. A content analysis is applied to a random sample of pieces news $(n=159)$ which follow these criteria: number of pieces about the crisis, duration, position on the broadcast, frame and kind of sources which appear on the news. It concludes that the main sensationalist feature in the sample is the preference towards individual and personal sources. Meanwhile, the presence of interest human frame is not significant.
\end{abstract}

Keywords:Television, economic crisis, sensationalism, infotainment, human interest, personalization

\title{
Introducción
}

En los últimos años la información televisiva en España y en Europa ha mostrado una clara tendencia hacia el infoentretenimiento (García Avilés, 2007). El auge de este género híbrido entre la información y el entretenimiento no se manifiesta exclusivamente en la proliferación de programas dedicados íntegramente a esta manera de presentar la actualidad (como por ejemplo, España Directo o Conexión Samanta) sino que también ha influido en los espacios clásicos de información televisiva (Ortell Badenes, 2014). Esta nueva tendencia está vinculada íntimamente al sensacionalismo (Kleemans y Hendriks Vettehen, 2009; Thussu, 2007).

Como parte de esta influencia los informativos televisivos han incorporado a su oferta televisiva una mayor proporción de noticias blandas frente a los contenidos significativos y relevantes de las noticias duras o hard news (Berrocal-Gonzalo et al, 2014). Las soft news se diferencian de las hard news en cuanto que los temas que abordan son políticamente irrelevantes, son narradas de forma episódica, se centran en las consecuencias individuales de los acontecimientos y su estilo es más personal y emocional (Reinemann et al, 2012). No obstante el amarillismo no se caracteriza tanto por el tipo de noticias sobre las que informa sino por el modo en que las presenta (Grabe, Zhou y Barnett, 2001). En este sentido el infoentretenimiento se define por favorecer el enfoque de interés humano en sus contenidos (Holtz-Bacha y Norris, 2001; Brants, 1998). Esta perspectiva presenta un acontecimiento, tema o problema desde un punto de vista emocional o centrándose en la historia de un individuo particular (Valkenburg, Semetko y de Vrees, 1999, p. 551).

El auge de este tipo de periodismo ha perjudicado seriamente la calidad de la información de la que disponen los ciudadanos. Se fomenta de este modo un 
conocimiento superficial de la realidad (Redondo García y Campos-Domínguez, 2015), parcial y sesgado (Marín Lladó, 2012) que desemboca en un mayor cinismo respecto a la agenda pública (Boukes y Boomgaarden, 2014).

Desde el campo académico se ha prestado especial atención a la relación entre infoentretenimiento e información política (p. ej., Abel \& Barthel, 2013; Nguyen, 2012), e incluso entre infoentretenimiento y guerra (p. ej., Paz \& Montero, 2011; Schwalbe, Silcock y Keith, 2008), pero no existen estudios que hayan abordado la relación entre este fenómeno y otro tipo de información considerada tradicionalmente "de calidad" (Calvo Gutiérrez, 2011): la información económica. Y ello pese a que la principal fuente de esta información para el público es precisamente la televisión (Goidel, Procopio, Terrell y Wu, 2010). Las noticias económicas han demostrado tener una amplia influencia tanto en las expectativas de la ciudadanía respecto al futuro de su país (Boomgaarden, van Spanje, Vliegenthart y de Vreese, 2011) como en los hábitos de consumo y ahorro de los ciudadanos (Martínez Fernández, Juanatey Boga y Costa Sánchez, 2012). La información negativa repercute en mayor grado sobre los públicos y los medios que las noticias positivas (Soroka, 2006; Goidel y Langley, 1995). Por ello -entre otros motivos- los estudios académicos en Comunicación han mostrado tradicionalmente un fuerte interés por la cobertura mediática de las crisis económicas (vid. p.ej.: Blood y Philips, 1995; Wu, Stevenson, Chen y Guner, 2002). No obstante los estudios sobre la cobertura mediática de la actual recesión se hallan todavía en una fase inicial (Schifferes y Coulter, 2013) y no existe consenso acerca del papel desempeñado por los medios y su información económica (Arrese y Vara-Miguel, 2014).

\section{Objetivo e hipótesis}

El objetivo de esta investigación consiste en medir el impacto del infoentretenimiento en la información televisiva dedicada a la crisis económica en los espacios informativos de Cuatro.

Para ello se enuncian las siguientes hipótesis:

H1: La atención de telediario de Cuatro a la crisis económica fue marginal.

Se parte del supuesto de que los informativos de televisión concedieron más importancia a otro tipo de noticias clasificables como soft news o noticias blandas que a los contenidos relativos a la crisis económica.

Para abarcar de manera más amplia el concepto de atención informativa esta hipótesis se ha dividido en las tres subhipótesis siguientes: 


\section{H1.1: Los informativos de Cuatro ofrecieron escasas noticias sobre la crisis}

Esta subhipótesis hace referencia al número de noticias sobre la crisis económica que incluyó cada informativo y al porcentaje de la duración que ocuparon respecto al resto de contenidos. Se considera que una ratio inferior al 10\% sería un nivel de interés bajo.

H1.2: La información sobre la crisis apareció en lugares secundarios de los informativos de Cuatro

Se ha considerado igualmente que la duración de las noticias no es el único factor por el que medir la importancia concedida a la crisis económica. De acuerdo con Canel e Innerarity (2000) se ha tenido en consideración la posición que ocupa la noticia en la escaleta. Se espera que las noticias sobre la crisis aparecieron en la segunda mitad del informativo.

H1.3: La información sobre la crisis en los informativos de Cuatro se ofreció fundamentalmente en formatos breves.

La última de estas subhipótesis plantea que la información sobre la crisis apareció de manera más frecuente a modo de piezas breves, inferiores a un minuto. Se parte del supuesto de que las noticias o temas más relevantes tienden a ofrecerse en piezas de mayor extensión. Se espera por lo tanto que la información de la crisis no supere una duración de 60 segundos.

H2: La cobertura de la crisis económica adoptó preferentemente un encuadre de "interés humano"

Dado que el infoentretenimiento se caracteriza por una fuerte presencia del encuadre del interés humano se ha intentado identificar las distintas perspectivas desde la que se presenta la actualidad. Se propone averiguar de este modo si la información emitida por Cuatro sobre este asunto adoptó una perspectiva de interés humano o si por el contrario ofreció encuadres para explicar y contextualizar la información.

H3: La principal fuente de información fue el testimonio de los propios ciudadanos

Otra de las características básicas del sensacionalismo televisivo y del infoentretenimiento consiste en la personalización de las noticias (Hendricks Vettehen, Nuijten y Beentjes, 2005). Como síntoma de esta hipótesis se ha empleado el criterio de la aparición de entrevistas a individuos que relatan su experiencia personal o dan su opinión acerca del tema sobre el que habla la noticia (Hendricks Vettehen, Nuijten, y Peeters, 2008). Por ello se espera que las principales fuentes que aparecieron en las noticias sobre la crisis en estos informativos respondan a esta categoría. 


\section{Objeto de estudio y métodos}

Este trabajo aplica su análisis sobre una muestra aleatoria de noticias sobre la crisis emitidas en un periodo concreto de 2012 por los informativos de una de las más importantes cadenas privadas de España: Cuatro ${ }^{1}$.

Para conseguir la selección aleatoria se eligió por sorteo una muestra de 21 días diferentes no correlativos repartidos entre la presentación de los presupuestos generales del Estado el 2 de abril de 2012 y la semana anterior a la aprobación del plan de rescate bancario acordado el 9 de junio del mismo año. De cada día se incluyeron tanto los informativos emitidos al mediodía como los de horario de acceso a primetime. Esta aleatoriedad ha abarcado tanto telediarios emitidos en días laborables (27 de ellos) como en fines de semana y festivos (14 de ellos).

La siguiente selección tuvo lugar entre las noticias emitidas en estos informativos. Dado que el objeto concreto del estudio consiste en analizar la cobertura del asunto específico de la crisis económica se ha tratado de incluir únicamente aquellas piezas referidas a este aspecto de la actualidad informativa. Siguiendo otros estudios (DíazCampo y Segado, 2015; Gold et al, 2011) se elaboró un listado de términos cuya presencia sirve como criterio para su inclusión en la muestra final. Todos estos criterios están asociados al concepto genérico y hasta cierto punto ambiguo de "crisis económica" ${ }^{2}$. Este listado se elaboró mediante una observación de los telediarios analizados y a partir de los resultados de una serie de entrevistas en profundidad realizadas de manera preliminar al estudio con un grupo de sociólogos, comunicadores y economistas.

Se emplea el análisis de contenido puesto que se trata de una metodología de demostrada eficacia para contrastar la calidad y los posibles sesgos de la información televisiva sobre asuntos económicos en diversos estudios (vid. Bell y Entman, 2011, p. 563; Jackson, 2011). El código aplicado ha sido adaptado de Del Moral, Quesada, Sánchez Aranda, León y Fernández (2007). De acuerdo con esta herramienta se han identificado los siguientes tipos de encuadre:

- Enfrentamiento: se pretende resaltar lo conflictivo de la situación o el desencuentro entre los protagonistas de la noticia. (Ejemplo: Una noticia que muestra el profundo y tajante desacuerdo de los grupos políticos de oposición ante una medida del gobierno)

\footnotetext{
${ }^{1}$ En abril de 2012 Cuatro ocupaba un 5,9\% de share frente al 13\% de TVE1, al 13\% de Tele 5 y al 12,2\% de Antena 3, según datos de Kantar Media (antes TNSofres)

${ }^{2}$ La lista completa de términos empleados son: Recesión, Situación económica, Contracción, Racionalización del gasto, Recortes, Austeridad, Reformas, Rescate, Ajustes, Intervención, Medidas, Rebaja, Inyección, Deuda, Déficit, Ahorro y Liquidez
} 
- Conjetural: La información es narrada como posible, es decir, se especula con algunos elementos de la misma (Ejemplo: En una noticia sobre un asesinato se especula sobre la autoría)

- Histórico: La noticia se explica como un proceso que hunde sus raíces en acontecimientos anteriores. (Ejemplo: Explicación de un acontecimiento narrando su evolución desde el origen del mismo)

- Perspectivista: Hace referencia a las posibles repercusiones que un acontecimiento puede tener en el futuro. (Ejemplo: Se indican las consecuencias que puede tener en el consumo medio del español la subida del IVA)

- Consensual: Pretende hacer hincapié en los aspectos de entendimiento en relación a un acontecimiento concreto. (Ejemplo: los miembros del gobierno y de la oposición manifiestan su rechazo ante un atentado terrorista)

- Competitivo: Se subraya la primacía de un elemento frente a otro. (Ejemplo: una retransmisión deportiva)

- Denunciador: Se pone en relevancia la injusticia o el rechazo ante una determinada situación que se considera inadmisible. (Ejemplo: la visibilidad de las víctimas de algunos de los casos abiertos sobre niños robados)

- Documental: Pretende mostrar de forma neutra la noticia introduciendo todo tipo de fuentes (entrevistas, clips de imágenes...) que aporten datos.

- Reactivo: Recoge la respuesta de diferentes grupos ante un acontecimiento determinado. (Ejemplo: tras un partido de fútbol se pregunta a los miembros y la directiva de ambos equipos cómo han seguido la competición)

- Interés humano: Pretenden hacer hincapié en el punto de vista emotivo y emocional de la noticia (Ejemplo: el desarrollo de nuevas tecnologías ha permitido que una persona enferma sane y lleve una vida normal)

Las fuentes utilizadas se han considerado un elemento especialmente importante para describir las noticias. Se ha establecido una clasificación atendiendo al origen de la misma, aunque se ha partido de la idea de que una noticia puede construirse a partir de fuentes diversas. De entre las fuentes personales se ha diferenciado entre:

- Implicada/Interesada: Se acude a persona directamente relacionadas y afectadas por la noticia y que tienen, por tanto, una visión claramente subjetiva de la cuestión (Ejemplo: trabajador despedido de una empresa que acaba de hacer un ERE)

- Encuestados: ciudadanos anónimos a los que se pregunta su opinión sobre el tema del que se está informando.

El resto de fuentes han sido clasificadas como: 
- Originaria: la fuente a la que se ha acudido es de donde parte la noticia (Ejemplo: Un comunicado oficial del gobierno anunciando una medida...)

- Consultada: el medio de comunicación busca introducir otra opinión o punto de vista a través de la presencia de una fuente que puede añadir profundidad o contraste a la información dada. (Ejemplo: tras un asesinato de género, el medio aporta testimonios que comentan la relación que tenía la pareja)

- Equilibradas: Se busca el testimonio de todas las partes implicadas en el acontecimiento (Ejemplo: opinión de un trabajador despedido por el ERE y el comentario de la empresa que he llevado a cabo el despido)

- Experta: El medio acude a un experto en la materia que esté tratando para explicar en profundidad la noticia dada. (Ejemplo: testimonio de un oceanógrafo tras un tsunami)

- Implícitas: En el caso de que no se mencione específicamente una fuente para la información

La codificación ha sido llevada a cabo por un único investigador, por lo que no ha resultado necesario calcular ninguna prueba de fiabilidad como el alfa de Cronbach

Este estudio forma parte de un proyecto conjunto sobre la representación mediática de la crisis económica en los informativos televisivos que también ha abordado la cobertura de este asunto en otras televisiones privadas -concretamente Tele $5 \mathrm{y}$ Antena 3-.

\section{Resultados}

\subsection{Atención mostrada a la crisis económica}

Durante el periodo estudiado los informativos de prime time de Cuatro emitieron un total de 159 noticias sobre la crisis económica. Esta cifra implica que la escaleta de cada telediario incluyó una media de 7,9 noticias sobre una media de 33,75 noticias por informativo. En términos de extensión temporal, de los 38:51 minutos de duración media de cada edición del informativo, 8:45 se dedicaron a cubrir la actualidad de la crisis. Es decir, el 22,52\% del tiempo de emisión se ha ocupado de la crisis. Más de una quinta parte de la escaleta de cada informativo ha estado volcada en esta faceta de la actualidad. 
La presencia de la crisis en el repertorio temático de Cuatro es constante puesto que en todos los informativos analizados se han emitido piezas sobre la cuestión. El Noticias Cuatro que menos noticias emitió sobre la crisis fue el del 13 de abril, que únicamente trató este tema en tres de sus informaciones. Por el contrario el día con una mayor presencia del tema fue el 12 de mayo, con 14 noticias sobre la crisis.

Por lo tanto la hipótesis H1.1 ("Los informativos de Cuatro ofrecieron escasas noticias sobre la crisis") queda refutada

El segundo gran criterio de esta hipótesis consistía en comprobar el grado de importancia concedido a las noticias atendiendo a su puesto en la escaleta. En este caso concreto el puesto de emisión medio de estas noticias se sitúa entre el octavo y el noveno lugar, concretamente en un hipotético puesto 8,75. Teniendo en cuenta que, como ya se ha especificado, la media de los informativos de la cadena estuvo compuesto por una media 33,75 noticias se puede señalar que la mayor parte de las noticias emitidas sobre la crisis se concentró en el primer tercil de la escaleta.

Además el $20,13 \%$ de estas noticias se incluyeron en titulares. El 79,87\% restante se trata de piezas que se desarrollan con posterioridad a lo largo del informativo. Este hecho no implica que todas estas noticias se traten como asunto de segundo orden, puesto que el $43,4 \%$ de las piezas consistieron en desarrollos que ampliaban la información avanzada en titulares. Tan solo el 36,47\% de estas noticias se corresponde con casos de piezas cuyos aspectos no son tratados ni avanzados en titulares.

Así pues la hipótesis H1.2 ("La cobertura de la crisis económica adoptó preferentemente un encuadre de "interés humano") también queda refutada.

La tercera y última subhipótesis referida a la importancia concedida a la crisis en estos informativos (H1.3) sostenía que "La información sobre la crisis se ofreció fundamentalmente en formatos breves".

Los resultados del análisis de contenido demuestran que la duración media de cada pieza emitida sobre la crisis fue de 68 segundos. Sin embargo, esta media se debe a la existencia de piezas más largas aisladas que rozaban los 3 minutos. Estas se han encontrado el 15 y el 18 de abril. La primera de ellas consiste en una pieza sobre el posible rescate a España en la que diversas personalidades políticas y económicas desde Luis de Guindos o Miguel Ángel Fernández Ordóñez a Felipe González y Josep Antoni Duran i Lleida pasando por Mariano Rajoy- descartan la posibilidad de que el país fuese intervenido. La segunda de ellas consiste en una información sobre las medidas del gobierno para ahorrar en sanidad en la que la ministra Ana Mato explica otras decisiones complementarias al copago sanitario, como la persecución del turismo sanitario o la solicitud a las farmacéuticas para que reduzcan el número de medicamentos en cada envase para ajustarlo a las dosis de cada tratamiento. 
Por lo tanto, es necesario calcular la mediana y la moda de la duración de estas piezas. La moda, es decir la duración más habitual entre las noticias analizadas, es de 38 segundos. La mediana, entendida como el valor central de los datos de duración, se sitúa en 65 segundos. Por lo tanto, la hipótesis H1.3 no puede ser refutada ni comprobada totalmente. La importancia concedida a determinados acontecimientos concretos de la agenda informativa se traduce en que a estos episodios se les dedica noticias de mayor duración. Sin embargo, eliminados estos valores extremos la duración de las noticias a otros asuntos se reduce sensiblemente.

\subsection{Encuadres}

La segunda hipótesis sobre la que se construye este estudio (H2 "La cobertura de la crisis económica adoptó un encuadre preferentemente de interés humano") tampoco puede constar como validada. El análisis de los enfoques arroja que los más abundantes son los de tipo reactivo $(29,11 \%)$, conjetural $(25,32 \%)$ y de enfrentamiento $(20,89 \%)$.

Así pues las noticias de Cuatro sobre la crisis solieron centrarse en las respuestas generadas por las acciones anunciadas por el gobierno o por otros actores. Como ejemplo puede citarse una pieza emitida el 21 de abril sobre las manifestaciones en contra de los recortes en educación en Cataluña y en España, en la que se muestran las protestas por estas medidas. También abundaban las especulaciones sobre los posibles efectos o consecuencias de estas reformas o la toma de otro tipo de medidas como pudiera ser la solicitud de un rescate financiero o la aplicación de nuevos recortes. Así ocurre por ejemplo el 16 de mayo con una pieza en la que Mariano Rajoy desmiente la posibilidad de que en España vaya a ocurrir un "corralito" como en Argentina. Finalmente el tercer gran enfoque presente en estos informativos fue el que presentaba el enfrentamiento entre gobierno y oposición y otros sectores sociales a raíz de las medidas impuestas ante la crisis. Este hecho no debería resultar extraño puesto que la polémica, la controversia y el enfrentamiento en definitiva son valores noticiosos clásicos (cf. Díaz Rojo, 2009)

El enfoque de interés humano aparece en un 17,09\% de las ocasiones. Este tipo de enfoque se empleaba para presentar de manera amable algún tipo de iniciativa individual o de pequeños colectivos ante la crisis. De este modo una noticia del 15 de abril habla del encuentro de un grupo de programadores de videojuegos que intentan vender sus últimas creaciones. Este tipo de noticias solían ubicarse en el último tramo de la escaleta del informativo.

Por el contrario, los encuadres que más podrían contribuir a una mejor comprensión de la crisis por parte del espectador fueron menos empleadas. Concretamente el 
enfoque documental se aplicó en un 15,19\% de ocasiones y apenas una de cada cinco noticias sobre la crisis empleó un enfoque de tipo histórico (un 5,06\%, exactamente).

\subsection{Personalización}

Finalmente los datos obtenidos en el análisis de contenido permiten verificar la hipótesis H3 ("La principal fuente de información fue el testimonio de los propios ciudadanos"). Aproximadamente una de cada cuatro fuentes fue una fuente implicada. En un 25,95\% de las ocasiones apareció algún afectado por la información relatando su experiencia o dando su opinión. También posee una presencia relevante otro tipo de fuentes consideradas "personales": consultadas en un $14,56 \%$ de las ocasiones y encuestadas en un $8,96 \%$. Tan solo en un $5,06 \%$ de las noticias se recurrió a una fuente experta. Se comprueba de este modo el escaso interés de los informativos de Cuatro hacia expertos en la materia. Prefirieron acudir a otro tipo de personas e instituciones para ilustrar o enriquecer las noticias. Tan solo se recurrió a fuentes expertas en 8 noticias, es decir, en el $5 \%$ de las ocasiones. Por ejemplo, en una pieza emitida el 31 de mayo se emiten unas declaraciones de Alberto Cardono, abogado de Ausbanc, en las que explica el funcionamiento de las participaciones preferentes para ilustrar una información sobre el fallo de un juzgado a favor de los afectados por este producto bancario.

\section{Conclusiones}

La influencia del infoentretenimiento y el amarillismo en las noticias sobre la crisis emitidas por Cuatro fue especialmente patente en el caso de la selección de las fuentes donde se muestra una clara preferencia por el recurso a personas individuales tal y como acaba de exponerse.

El impacto del sensacionalismo sobre el tipo de encuadre escogido es menos relevante. Se ha comprobado que este enfoque no disfrutó de especial preponderancia sobre otro tipo de perspectivas. No obstante, esto no implica que la los enfoques adoptados por las noticias de la cadena de Mediaset fuesen de tipo más explicativo o didáctico.

Sin embargo, como se apuntó en la introducción, estudios previos sí que han destacado la mayor preferencia de las noticias sobre política por el interés humano. Esta diferencia entre información económica e información política puede deberse en primer lugar a la propia naturaleza del tema abordado. Dentro de un contexto económico, político y social marcado por la recesión la banalización de la realidad 
económica parece tener menos cabida en este repertorio temático que en otros asuntos.

De manera simétrica, la misma explicación puede aplicarse a la importancia concedida a la información sobre la crisis. Pese a la influencia que el infoentretenimiento y el amarillismo ejercen sobre la agenda temática a favor de las noticias blandas la situación económica percibida por los ciudadanos provocó una demanda de noticias sobre esta coyuntura. Resultaría poco natural y quizá contraproducente sustituir noticias sobre este asunto por un repertorio de soft news.

Entre las limitaciones del estudio cabe mencionar que solo se han analizado las noticias de un canal concreto de televisión, por lo que es necesario relativizar la universalidad de los resultados. Los datos obtenidos deberían ser entendidos exclusivamente en contexto con los resultados que estudios similares pueden arrojar de otras cadenas. Además, el relativamente bajo nivel de audiencia conseguido por este canal de televisión puede rebajar sensiblemente la representatividad de los datos.

No obstante esta investigación permite señalar de modo exploratorio las distintas maneras en que el infoentretenimiento y el sensacionalismo en televisión han afectado a una categoría especializada de noticias: las de tipo económico. Como parte de futuros estudios planteables a partir de este momento surge la necesidad de comparar este tratamiento informativo en otras cadenas televisivas durante el mismo periodo o en periodos posteriores.

\section{Referencias bibliográficas}

ABEL, A. D., \& BARTHEL, M. (2013). "Appropriation of Mainstream News: How Saturday Night Live Changed the Political Discussion". Critical Studies in Media Communication, 30 (1), 1-16.

ARRESE, Á., \& VARA-MIGUEL, A. (2014). “Alarma y alarmismo: medios de comunicación y crisis económica". Estudios sobre el Mensaje Periodístico, 20 (2), 933-851.

BELL, C. V., \& ENTMAN, R. M. (2011). "The media's role in America's exceptional politics of inequality". The International Journal of Press/Politics, 16 (4), 548572.

BERROCAL GONZALO, S. REDONDO GARCÍA, M., MARTÍN JIMÉNEZ, V. \& CAMPOS DOMÍNGUEZ, E. (2014). "La presencia del infoentretenimiento en los canales generalistas de la TDT española". Revista Latina de Comunicación Social [en línea], 69, 85-

103. http://www.revistalatinacs.org/069/paper/1002 UVa/05 Be.html (12-62016) 
BLOOD, D. J. \& PHILLIPS, P. C. B. (1995). “Recession headline news, consumer sentiment, the state of the economy and presidential popularity. A time-series analysis 1989-1993". International journal of public opinion, 7 (1), 1-22

BOOMGAARDEN, H. G., VAN SPANJE, K., VLIEGENTHART, R., \& DE VREESE, C. H. (2011). "Covering the crisis: Media coverage of the economic crisis and citizens' economic expectations". Acta Politica, 46 (4), 353-379.

BOUKES, M., \& BOOMGAARDEN, H. G. (2014). "Soft News With Hard Consequences? Introducing a Nuanced Measure of Soft Versus Hard News Exposure and Its Relationship With Political Cynicism". Communication Research, 42 (5), 701-731

BRANTS, K. (1998). "Who's afraid of infotainment?". European Journal of Communication, 13 (3), 315-335.

CANEL, M. J. \& INNERARITY, C. (2000). “La campaña electoral en la television”. En: A. Martínez y M. Méndez (eds). Las elecciones al Parlamento Europeo, Valencia: Tirant lo Blanch, 171-190

CALVO GUTIÉRREZ, E. (2011). “La supervivencia del gran periodismo financiero". Fonseca Journal of Communication, 2, 55-70

DEL MORAL, J., QUESADA, M., SÁNCHEZ, J., LEÓN, B. \& FERNÁNDEZ, A. (2007). El análisis de la información televisiva: hacia una medida de la calidad periodística. Madrid: CIE Dossat.

DÍAZ-CAMPO, J., \& SEGADO-BOJ, F.(2015). "Journalism ethics in a digital environment: How journalistic codes of ethics have been adapted to the Internet and ICTs in countries around the world". Telematics and Informatics,32 (4), 735-744.

DÍAZ ROJO, J. A. (2009). Los valores noticiosos como práctica discursiva periodística. Espéculo: Revista de Estudios Literarios [en línea], (41), 95. http://pendientedemigracion.ucm.es/info/especulo/numero41/valonoti.html (15-07-2015)

GARCÍA AVILÉS, J. A. (2007). "El infoentretenimiento en los informativos líderes de audiencia en la Unión Europea”. Anàlisi, 35, 47-63.

GARCÍA REDONDO, M. \& CAMPOS-DOMÍNGUEZ, E (2015). "Implicaciones éticas del infoentretenimiento televisivo". Comunicació: Revista de Recerca i d'Anàlisi, 32 (1), 73-89.

GOIDEL, R. K. \& LANGLEY, R. E. (1995). “Media coverage of the economy and aggregate economic evaluations. Uncovering evidence of indirect media effects". Political Research Quarterly, 48 (2), 313-328.

GOLD, J., PEDRANA, A. E.; SACKS-DAVIS, R., HELLARD, M. E.; CHANG, S., HOWARD, S., KEOGH, L., HOCKING, J. S.; STOOVE, M. A. (2011). "A systematic examination of the use of online social networking sites for sexual health promotion" [en línea]. BMC Public Health, 11. http://www.biomedcentral.com/content/pdf/1471-2458- 11-583.pdf (12-62015)

HENDRICKS VETTEHEN, P., NUIJTEN, K., \& BEENTJES, J. (2005). "News in an age of competition: The case of sensationalism in Dutch television news, 1995-2001". Journal of Broadcasting \& Electronic Media, 49 (3), 282-295.

HENDRICKS VETTEHEN, P., NUIJTEN, K., \& PEETERS, A. (2008). "Explaining effects of sensationalism on liking of television news stories: The role of emotional arousal". Communication Research, 35 (3), 319-338. 
HOLTZ-BACHA, C., \& NORRIS, P. (2001). "To entertain, inform and educate: Still the role of public television". Political Communication, 18 (2), 123-140.

KLEEMANS, M., \& HENDRIKS VETTEHEN, P. (2009). Sensationalism in television news: A review. En Konig, R. P., Nelissen, P. W. M. \& Huysmans, F. J. M. (Eds.), Meaningful media: Communication research on the social construction of reality. Nijmegen: Tandem Felix, 226-243

JACKSON, D. (2011). "Strategic news frames and public policy debates: Press and television news coverage of the euro in the UK". Communications - European Journal of Communication Research, 36 (2), 169-193.

MARÍN LLADÓ, C. M. (2012). "La pérdida de objetividad en las noticias de los programas de infoentretenimiento en televisión". AdComunica, (4), 81-98.

MARTÍNEZ FERNÁNDEZ, V. A., JUANATEY BOGA, Ó., COSTA SÁNCHEZ, C. (2012). "Agenda setting y crisis económica: Influencia de la prensa en el comportamiento de consumo y ahorro". Estudios sobre el mensaje periodístico, 18 (1), 147-156.

NGUYEN, A. (2012). "The effect of soft news on public attachment to the news: Is 'infotainment' good for democracy?" Journalism Studies, 13 (5-6), 706-717.

ORTELLS-BADENES, S. (2014). "Los criterios de noticiabilidad periodística en los programas de infoentretenimiento". Textual \& Visual Media, 7, 2014, 207-220.

PAZ, M. A. \& MONTERO, J. (2011). "Las profecías son noticia. El uso del futuro en la información televisiva española sobre la crisis de Irak". Comunicación y Sociedad, 23 (1), 153-174.

REINEMANN, C., STANYER, J., SCHERR, S., \& LEGNANTE, G. (2012). "Hard and soft news: A review of concepts, operationalizations and key findings". Journalism, 13 (2), 221-239.

SCHIFFERES, S., \& COULTER, S. (2013). "Downloading disaster: BBC news online coverage of the global financial crisis". Journalism, 14 (2), 228-252.

SCHWALBE, C. B., SILCOCK, W., \& KEITH, S. (2008). Visual Framing of the Early Weeks of the U.S.-Led Invasion of Iraq: Applying the Master War Narrative to Electronic and Print Images. Journal of Broadcasting \& Electronic Media, 52 (3), 448-465.

SOROKA, S. N. (2006)." Good news and bad news: Asymmetric responses to economic information". Journal of Politics, 68 (2), 372-385.

THUSSU, D. K. (2007). News as entertainment. The rise of global infotainment. Londres: Sage.

VALKENBURG, P., SEMETKO, H. \& DE VREESE, C. (1999). "The effects of news frames on reader's thoughts and recall". Communication Research, 26 (5), 550-569.

WU, H. D., STEVENSON, R. L., CHEN, H. C., GUNER, Z. N. (2002). "The conditioned impact of recession news: A time-series analysis of economic communication in the United States, 1987-1996". International Journal of Public Opinion, 14 (1), 19-36. 